\title{
Creatividad e Inteligencias Múltiples según el género en alumnado de Educación Primaria
}

María Pilar Garín Vallverdú, Verónica López Fernández y Fátima Llamas Salguero. Universidad Internacional de la Rioja

Recepción: 22 de febrero de 2016 | Revisión: 24 de febrero de 2016 | Aceptado: 25 febrero de 2016

Correspondencia: veronica.lopez@unir.net

Citar: Garin-Vallverdu, M.P., Lopez-Fernandez, V. y Llamas-Salguero, F. (2016). Creatividad e Inteligencias Múltiples según el género en alumnado de Educación Primaria. ReiDoCrea, 5, 33-39.

Resumen: Los primeros años de escolarización son fundamentales para potenciar el desarrollo de la creatividad y las inteligencias múltiples. Bajo el sustento de los marcos teóricos de referencia que explican ambas variables, el objetivo del estudio fue analizar las diferencias significativas existentes en la creatividad y las inteligencias múltiples entre niños y niñas de $1^{\circ}$ de Educación primaria. La muestra se compuso de 60 estudiantes (chicos $=31$; chicas $=29$ ) de 6 y 7 años escolarizados en un centro público de Zaragoza. Los instrumentos utilizados fueron el Cuestionario del profesorado para diagnosticar Inteligencias Múltiples en Primaria y el Test de Pensamiento Creativo de Torrance. Los resultados mostraron diferencias significativas en la creatividad, siendo las chicas quienes obtuvieron mayores puntuaciones. Respecto a las inteligencias múltiples, la inteligencia interpersonal fue la que mayores puntuaciones obtuvo en ambos géneros. Paralelamente, la inteligencia musical fue la de menor puntuación en ambos géneros, siendo la única que mostró diferencias significativas. Estos hallazgos se discuten argumentando la necesidad de realizar un programa de intervención que potencie la creatividad y la inteligencia musical en ambos géneros a través del resto de inteligencias, significando un aprendizaje transversal que optimice la formación del alumnado de manera íntegra.

Palabras clave: Creatividad | Inteligencias Múltiples | Género

Creativity and Multiple Intelligences Students by Gender in Primary Education

\begin{abstract}
The early years of schooling are considered fundamental for an optimal development of creativity and multiple intelligences in both boys and girls. Using theoretical frameworks that explain both variables, the aim of this study is to analyze the significant differences in creativity and multiple intelligences between First Grade students. The sample consisted of 60 students (boys $=31$, girls $=29$ ) aged between 6 and 7 years belonging to a public school in Zaragoza. The instruments used were the Torrance Creative Thinking Test and the questionnaire for teachers to diagnose Multiple Intelligences in Primary. The results showed significant differences in creativity, the girls obtaining higher scores. Regarding multiple intelligences, interpersonal intelligence which that which obtained higher scores in both sexes. Concurrently, musical intelligence scored lowest in boys and girls, being the only one that showed significant differences. These findings are discussed arguing the need for an intervention program that strengthens the development of creativity and musical intelligence in both sexes by way of the other intelligences and teamwork. This can mean cross-curricular learning to optimize student development in a holistic fashion.
\end{abstract}

Keywords: Creativity | Multiple Intelligences | Gender

Hoy en día, el sistema educativo orienta gran parte de sus esfuerzos y recursos a la obtención de altas puntuaciones y la mejora del rendimiento académico del alumnado. Esto conlleva que se deje de lado el desarrollo de otros procesos, como el de la creatividad, que se consideran necesarios para un óptimo aprendizaje en la infancia, sin tener en cuenta las características, necesidades, gustos e intereses de los estudiantes, frustrando la realización de sus tareas escolares. Esta desmotivación es considerada un factor detonante del fracaso escolar (Escudero, 2005), provocando un efecto paraguas que acoge múltiples realidades del alumnado. Por ello, la adaptación de la labor docente para el afrontamiento de estas necesidades e intereses de los estudiantes, ha de suponer un objetivo principal y prioritario en el colectivo de maestros/as.

La creatividad, influenciada por diversos factores, es una de las características que acompañan a las personas durante su desarrollo vital (Monreal, 2000). La escuela, es considerada un lugar idóneo para potenciarla debido a la flexibilidad cognitiva de los alumnos. En esta línea, Blázquez (2009) señala la relevancia de educar desde la creatividad, debido a que garantiza que nuestros alumnos se formen íntegramente para afrontar de manera eficaz los diferentes problemas que se les presente. Sin 
embargo, existen evidencias científicas que demuestran que las metodologías de trabajo adoptadas por la mayoría del profesorado en las aula está frustrando en gran medida su desarrollo entre los estudiantes, debido a su carácter fuertemente sistemático y mecanizado (Coll, 1999).

Una teoría reciente que sustenta el presente estudio es en la Teoría de las Inteligencias Múltiples de Gardner $(1987,1993)$, la cual sostiene que cada persona posee las ocho inteligencias (Inteligencia Lingüística, Inteligencia Lógico-Matemática, Inteligencia Espacial, Inteligencia Corporal-Cinestésica, Inteligencia Musical, Inteligencia Naturalista, Inteligencia Interpersonal e Inteligencia Intrapersonal) y que juntas funcionan de manera única en cada persona. Además, este marco teórico hace hincapié en la rica diversidad con la que las personas demuestran sus habilidades dentro de cada inteligencia y entre ellas (Armstrong, 2011). De aquí se desprende que no todos los alumnos son iguales, teniendo cada uno sus propias particularidades y necesidades educativas, por lo que es necesario plantear nuevos métodos de aprendizaje que se ajusten a esas características. En este sentido, parece necesaria la identificación de las potencialidades y debilidades de cada tipo de inteligencia en el alumnado. Todo ello, permitirá realizar un programa de intervención basado en evidencias científicas que priorice el desarrollo de los puntos débiles de los estudiantes a través de sus puntos fuertes, el cual optimizaría el desarrollo íntegro de la persona.

Con este trabajo se pretender fomentar la creatividad y las diversas inteligencias múltiples mediante un programa de intervención realizado a través de la identificación de la realidad de aula vivida en un centro educativo de primaria. Esta intervención está basada en el aprendizaje cooperativo con el fin de atender las particularidades y necesidades de cada uno de los estudiantes.

\section{Método}

\section{Participantes}

La población accesible al estudio fueron un total de 76 estudiantes, con edades comprendidas entre los 6 y 7 años de edad, correspondientes a $1^{\circ}$ de Educación Primaria de un Colegio Público de Educación Infantil y Primaria de un centro urbano. Dicho centro se ubica en la zona centro de un Zaragoza, España. Se trata de un colegio bilingüe inglés, adscrito al currículum agrupado del British Council. El nivel socio-económico de las familias de los alumnos de este centro es medio-alto. En lo referente al primer curso, todos los niños son de nacionalidad española y han cursado Educación Infantil, excepto una alumna de nacionalidad rumana que se incorporó el último curso de Educación Infantil. Para poder participar en el estudio los alumnos tenían que cumplir con una serie de criterios de inclusión, los cuales fueron el consentimiento por parte de los padres y la adecuada realización de los cuestionarios. Así, tras aplicar dichos criterios se obtuvo una muestra total de 60 estudiantes, 31 de ellos de género masculino y 29 del género femenino, para el análisis de las inteligencias múltiples. Para el análisis de la creatividad, por no cumplir uno de los criterios de inclusión (no responder adecuadamente a la prueba de creatividad), un sujeto fue eliminado del estudio, obteniendo una muestra para esta variable de 59 estudiantes (chicos=31; chicas=28). 


\section{Instrumentos}

Las variables dependientes de este estudio son la creatividad y las inteligencias múltiples, mientras que el género del alumnado es la única variable independiente. Para medir dichas variables, se han aplicado los siguientes instrumentos:

- Cuestionario del profesorado para diagnosticar Inteligencias Múltiples en Primaria. Se ha utilizado la versión traducida al castellano de este instrumento, diseñado por Armstrong (2001). Este cuestionario se compone de ocho factores que responden a las ocho inteligencias múltiples. Cada factor está integrado por 10 ítems (e.g., "Piensa de una forma abstracta o conceptual superior al resto"). La escala de respuesta consta de tres opciones, sí (1 punto), no (0 puntos) o a veces ( 0.5 puntos) en función de las particularidades, actitudes, intereses y comportamientos de los sujetos evaluados. La puntuación se calcula a través de la suma de los 10 ítems de cada factor de manera independiente para cada una de las inteligencias

- Test de Pensamiento Creativo de Torrance (TTCT). Este instrumento el cual responde al acrónimo inglés Torrance Thinking of Creative Test, desarrollado por Torrance $(1966 ; 1974 ; 1984 ; 1990 ; 1998)$ se compone de dos partes independientes: una verbal y otra figurativa. En el presente estudio se ha aplicado la parte figurativa de manera individual, cuyo propósito es evaluar las producciones de los alumnos. Esta parte está formada a su vez por tres subpruebas: la primer a es la composición de un dibujo a partir de un trozo de papel verde, la segunda consiste en acabar 10 dibujos a partir de unos trazos ya dibujados, los cuales han de servir como referencia, y la tercera y última consiste en hacer tantos dibujos como se pueda a partir de 30 pares de líneas paralelas.

\section{Procedimiento}

En primer lugar, se contactó con el centro escolar para pedir la colaboración con este estudio a través de la aplicación de las pruebas. Seguidamente, se elaboró junto al equipo directivo una circular que autorizara, por parte de los padres, la aplicación de las pruebas mencionadas a los alumnos de $1^{\circ}$ de Educación Primaria, así como se acordó la colaboración de las tutoras correspondientes a dicho curso para la cumplimentación del cuestionario de inteligencias múltiples. Una vez recogidos los consentimientos de participación de los estudiantes firmados por sus padres, se comenzó el estudio.

Para realizar la aplicación de las pruebas de creatividad, se utilizó el aula de clase habitual de cada grupo en un ambiente óptimo de trabajo con la presencia de la responsable del presente estudio. El tiempo asignado para ello fue de una hora y quince minutos para realizar la prueba completa, contando con la explicación de cada una de las tres partes, la resolución de las dudas surgidas y el tiempo asignado para su ejecución (10 minutos cada una). En cuanto a los cuestionarios de inteligencias múltiples, fueron completados por cada una de las tutoras correspondientes a los diferentes grupos del centro que englobaban a los alumnos de la muestra del presente estudio.

Finalmente, se procedió a la evaluación y ponderación de los cuestionarios y pruebas aplicadas para su correspondiente análisis estadístico, obtención de resultados y extracción de conclusiones. 


\section{Análisis de datos}

Para comprobar el objetivo general del estudio además de los correspondientes específicos, y así, poder orientar la intervención posterior con evidencias científicas, se calcularon los estadísticos descriptivos (Media y Desviación Típica). Para comprobar si existían diferencias significativas en función del género en la creatividad y en las inteligencias múltiples se realizaron los correspondientes análisis de diferencias a través del estadístico $t$-student para muestras independientes (chicos; chicas). Todos estos cálculos se realizaron a través del programa estadístico SPSS-20.

\section{Resultados}

Los análisis descriptivos para los estudiantes de $1^{\circ}$ de Educación Primaria en referencia a la variable creatividad muestran medias aparentemente dispares en función del género. En este sentido, como se puede observar en las tabla 1, las chicas obtienen una media $(M=75.21)$ superior a la de los chicos $(M=61.84)$.

\begin{tabular}{|lcccccc|}
\hline \multicolumn{6}{|c|}{ Tabla 1. Análisis descriptivos de la creatividad en función del género } \\
\hline & & $\mathrm{n}$ & $\mathrm{M}$ & $\mathrm{DT}$ & Mín. & Máx. \\
\hline Creatividad & Chicos & 31 & 61.84 & 21.55 & 24 & 96 \\
& Chicas & 28 & 75.21 & 19.62 & 20 & 99 \\
\hline
\end{tabular}

En base a los factores que componen la variable de Inteligencias Múltiples, los análisis descriptivos en los estudiantes en función del género presentan las siguientes características que se pueden observar en la tabla 2. En referencia a la Inteligencia Lingüística las chicas obtienen una media $(M=6.00)$ mayor a la de los chicos $(M=5.05)$. Por el contrario, en la Inteligencia Lógico-Matemática son los chicos $(M=5.23)$ quienes tienen una media superior. De igual modo, en la Inteligencia Espacial, los chicos $(M=5.82)$ poseen una media superior a la de las chicas $(M=5.76)$, aunque en este caso las diferencias entre géneros son aparentemente residuales. Respecto a la Inteligencia Corporal-Cinéstesica, son también los chicos $(M=.5 .27)$ quienes obtienen una media superior, con una diferencia de .30 respecto a las chicas $(M=4.97)$. Siguiendo con la Inteligencia Musical, observando la tabla 2, se aprecia como son las chicas $(M=4.53)$ quienes obtienen valores notablemente mayores respecto a los chicos (3.26). En cambio, en la Inteligencia Naturalista, son los chicos quienes obtienen una media superior con una diferencia de .51. Respecto a la Inteligencia Interpersonal, es el factor de las inteligencias múltiples que muestra medias más similares entre ambos géneros, con una diferencia de .01 únicamente superior a las chicas. Finalmente, en la Inteligencia Intrapersonal son las chicas $(M=5.53)$ quienes obtienen una media superior a los chicos $(M=4.98)$.

A modo de resumen, la tabla 2 muestra como las chicas obtuvieron medias superiores respecto al género opuesto en la inteligencia lingüística, musical, intrapersonal e interpersonal. Por el contrario, en la inteligencia lógico-matemática, espacial, corporalcenestésica y naturalista fueron los chicos quienes obtuvieron una media superior. 


\begin{tabular}{|c|c|c|c|c|c|c|}
\hline Inteligencias & & $\mathrm{N}$ & $\mathrm{M}$ & DT & Mín. & Máx. \\
\hline \multirow[t]{2}{*}{ Lingüística } & Chicos & 31 & 5.05 & 3.19 & 0 & 10 \\
\hline & Chicas & 29 & 6.00 & 2.94 & 1 & 10 \\
\hline \multirow[t]{2}{*}{ Lógico-Matemática } & Chicos & 31 & 5.23 & 2.73 & 2 & 10 \\
\hline & Chicas & 29 & 4.69 & 2.44 & 0 & 9 \\
\hline \multirow[t]{2}{*}{ Espacial } & Chicos & 31 & 5.82 & 2.01 & 2.5 & 9.5 \\
\hline & Chicas & 29 & 5.76 & 1.47 & 2.5 & 8 \\
\hline \multirow[t]{2}{*}{ Corporal-Cinestésica } & Chicos & 31 & 5.27 & 1.81 & 2 & 9 \\
\hline & Chicas & 29 & 4.97 & 1.47 & 2 & 8 \\
\hline \multirow[t]{2}{*}{ Musical } & Chicos & 31 & 3.26 & 1.12 & 1 & 7 \\
\hline & Chicas & 29 & 4.53 & 1.54 & 2 & 8 \\
\hline \multirow[t]{2}{*}{ Naturalista } & Chicos & 31 & 5.82 & 2.63 & 1 & 10 \\
\hline & Chicas & 29 & 5.31 & 2.38 & 1 & 10 \\
\hline \multirow[t]{2}{*}{ Interpersonal } & Chicos & 31 & 6.37 & 2.16 & 1 & 10 \\
\hline & Chicas & 29 & 6.38 & 2.45 & 2 & 10 \\
\hline \multirow[t]{2}{*}{ Intrapersonal } & Chicos & 31 & 4.98 & 2.25 & 1 & 9 \\
\hline & Chicas & 29 & 5.53 & 1.96 & 2 & 9 \\
\hline
\end{tabular}

En lo que concierne al análisis de diferencias en función del género, la creatividad muestra una diferencia de medias significativa favorable a las chicas $(t=-2.48 ; p<.05)$ como se puede observar en la tabla 3.

\begin{tabular}{|lcc|}
\hline \multicolumn{2}{|c|}{ Tabla 3. Análisis de diferencias de la creatividad en función del género } \\
\hline Variables & $t$ & $p$ \\
\hline Creatividad & -2.48 & 0.16 \\
\hline
\end{tabular}

En cuanto a la variable de Inteligencias Múltiples, como se puede observar en la tabla 7 , el análisis de diferencias, únicamente muestra diferencias significativas en el factor de Inteligencia Musical, siendo esta superior en el género femenino $(t=-3.69 ; p<.01)$.

\begin{tabular}{|lcc|}
\hline \multicolumn{3}{l}{ Tabla 7. Análisis de diferencias de las inteligencias múltiples en función del género } \\
\hline Variables & $t$ & $p$ \\
\hline Inteligencia Lingüística & -1.2 & .235 \\
Inteligencia Lógico-Matemática & .8 & .426 \\
Inteligencia Espacial & .14 & .889 \\
Inteligencia Corporal-Cinestésica & .72 & .473 \\
Inteligencia Musical & -3.69 & .000 \\
Inteligencia Naturalista & .79 & .433 \\
Inteligencia Interpersonal & -.01 & .989 \\
Inteligencia Intrapersonal & -1 & .318 \\
\hline
\end{tabular}

\section{Discusión}

Para abordar el objetivo del presente estudio siguiendo a los autores más representativos de la creatividad en el ámbito escolar y la Teoría de las Inteligencias Múltiples de Gardner (1989), se plantearon tres hipótesis.

En primer lugar, se postuló que la creatividad presentaría diferencias significativas en función del género. Atendiendo a los resultados del presente estudio, se puede afirmar que la hipótesis es cierta. En esta línea, los resultados de las presente investigación muestran coherencia con los estudios de Xiaoxia (1999), quien demostró diferencias significativas en relación al género, habiendo utilizado el mismo instrumento de evaluación que este estudio, aunque abordando las cuatro características de la creatividad, siendo los chicos quien destacaban en el factor flexibilidad y las chicas en 
fluidez verbal. Sin embargo, otras investigaciones que han evaluado la creatividad en la Educación Primaria no han hallado diferencias significativas entre estudiantes de distinto género (Espinosa, 2004). Esta incongruencia con los resultados del presente estudio, así como con Xiaoxia (1999), puede ser debido a que el instrumento realizado por Espinosa (2004) fue diferente. En este sentido, el conjunto de resultados encontrados en la literatura científica son distintos atendiendo al instrumento de evaluación de la creatividad, por lo que sería oportuno evaluar la creatividad con el instrumento más aceptado por la comunidad científica para la Educación Primaria.

En segundo lugar, se postuló que existirían diferencias significativas respecto a las inteligencias múltiples en función del género. Siguiendo los resultados obtenidos en este estudio, la segunda hipótesis no se confirma, debido a que únicamente presentan diferencias significativas una de las ocho inteligencias múltiples, la inteligencia musical. En el resto de inteligencias, a pesar de mostrar medias diferentes entre géneros, no se puede afirmar que estas fueran significativas. Estos resultados no muestran coherencia con los hallados en otras investigaciones donde la inteligencia musical no mostró diferencias significativas entre ambos géneros (Pérez et al., 2011).

Por último, se planteó que los chicos obtendrían puntuaciones superiores en la inteligencia lógico-matemática, mientras que las chicas obtendrían mayores puntuaciones en las inteligencias musical e interpersonal. Esta hipótesis se cumple parcialmente: por una lado se confirma que los chicos obtienen puntuaciones superiores en la inteligencia lógico-matemática y que las chicas obtienen valores notablemente mayores en la inteligencia musical; sin embargo, no se puede afirmar que las chicas obtengan mayores puntuaciones en la inteligencia interpersonal, pues las medias encontradas entre géneros son similares. Respecto al género masculino, estos resultados son congruentes con los hallados por Pérez et al. (2011), quien mostró igualmente mayores diferencias entre géneros para la inteligencia lógicomatemática, favorables a los chicos. Asimismo, Ferrándiz et al. (2008) demostraron en su estudio que el género masculino no obtiene mayor puntuación en la inteligencia lógico-matemática respecto al género femenino. Sin embargo, para el género femenino los resultados se contraponen, debido a que en otras investigaciones han hallado valores más elevados para las inteligencias interpersonal y naturalista. De esto se desprende, que el género masculino obtienen valores mayores en la inteligencia lógico-matemática, pero con los estudios llevados a cabo hasta el momento, no existe univocidad en cuanto a las inteligencias en las que el género femenino obtienes valores superiores en referencia al masculino.

Como conclusión principal del estudio se desprende la necesidad de potenciar la creatividad en Educación Primaria en ambos géneros, haciendo incidencia en los estudiantes de género masculino debido a que han obtenido unas puntuaciones notablemente inferiores que las chicas. Otra aportación relevante del estudio, es la necesidad de potenciar la inteligencia musical en ambos géneros, debido a que es la que menores puntuaciones obtuvo en referencia al resto de inteligencias. Asimismo, no parece necesario diferenciar la intervención para el desarrollo de las inteligencias múltiples por género, al no hallar apenas diferencias significativas. Teniendo en cuenta todo ello, se propone un programa de intervención que busca el desarrollo de la inteligencia musical en ambos género a través del resto de inteligencias y que potencia a su vez la creatividad, mediante el trabajo cooperativo del alumnado.

Referencias

Armstrong, T. (2001). Cuestionario del profesorado para diagnosticar Inteligencias Múltiples en Primaria. 
Blázquez, A. (2009). Educación y creatividad. Innovación y experiencias educativas. Art. 38. Granada: CSI-F Enseñanza Andalucía.

Coll, C. (1999). Psicología de la educación. Madrid: Alianza.

Escudero, J.M. (2005). Fracaso escolar, exclusión educativa: ¿de qué se excluye y cómo? Profesorado, revista de currículum y formación del profesorado, 1(1), 1-24.

Espinosa, J.C. (2004) Incidencia del género y la edad en la creatividad infantil. Perspectivas en Psicología, 1(1), 22-30.

Ferrándiz, C., Bermejo, R. Sainz, M., Ferrando, M. y Prieto, M.D. (2008). Estudio del razonamiento lógico-matemático desde el modelo de las inteligencias múltiples. Anales de Psicología, 24(2), 220-225.

Gardner, H. (1987). Estructura de la mente. La teoría de las Inteligencias Múltiples. México: FCE.

Gardner, H. (1993a). Inteligencias multiples: la teoría en la práctica. Barcelona: Paidós.

Gardner, H. (1993b). Inteligencias múltiples. Buenos Aires: Paidós.

Monreal, C. (2000). Qué es la creatividad. Madrid: Biblioteca nueva.

Oliveira, E., Almeida, L., Ferrándiz, C., Ferrando, M., Sainz, M. y Prieto, M. D., (2008). Test de pensamiento creativo de Torrance (TTCT): elementos para la validez de constructo en adolescentes portugueses. Psicothema, 21(4), 562-567

Pérez, E., Lescano, C., Heredia, D., Zalazar, P., Furlán, L. y Martínez, M. (2011). Desarrollo y análisis psicométricos de un inventario de autoeficacia para iteligencias múltiples en niños argentinos. Psicoperspectivas, 10(1), 169189.

Sánchez, M. (1991). Desarrollo de habilidades del pensamiento. Creatividad. México: Trillas.

Xiaoxia, A. (1999). Creativity and academic achievement an investigation of gender differences. Creativity Research Journal, 12(4), 329-337. 